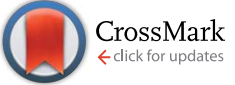

Cite this: RSC Adv., 2017, 7, 2127

Received 9th November 2016 Accepted 12th December 2016

DOI: $10.1039 / c 6 r a 26534 k$

www.rsc.org/advances

\section{Impact of mineral components in cow manure biochars on the adsorption and competitive adsorption of oxytetracycline and carbaryl $\dagger$}

\begin{abstract}
Mengwei Li, ${ }^{a}$ Zhendong Zhao, ${ }^{a}$ Xiaodan $\mathrm{Wu}{ }^{\mathrm{c}}$ Wenjun Zhou ${ }^{\star a b}$ and Lizhong Zhu ${ }^{\mathrm{ab}}$
In recent years, biochar has been well-documented in the literature as a promising soil amendment for the sorption and immobilization of organic contaminants in contaminated soils via its organic functional groups. In this study, original and demineralized cow manure biochars were applied with the aim to evaluate the impact of their mineral component on adsorption and competitive adsorption of oxytetracycline and carbaryl. Adsorption increased greatly for oxytetracycline but decreased slightly for carbaryl after demineralization. Mineral components significantly enhanced overall adsorption of carbaryl but slightly inhibited that of oxytetracycline to biochars, because the dominant sorption mechanisms of oxytetracycline and carbaryl on demineralized biochars were established as pore-filling effects and specific-site sorption, respectively. Competitive adsorption was observed in the binary-solute sorption system, where oxytetracycline outcompeted carbaryl on original biochars, however, carbaryl showed a greater competitive adsorption to oxytetracycline on demineralized biochars. Furthermore, demineralization treatment weakened the competition strength of oxytetracycline to carbaryl but enhanced that of carbaryl to oxytetracycline. This work demonstrated that the demineralization treatment altered the physicochemical properties of biochars and the dominant sorption mechanisms of oxytetracycline and carbaryl, leading to their different changes on competition strength. The results are useful for the application of biochars in the remediation of combined pollution in agricultural soils.
\end{abstract}

\section{Introduction}

The contamination of farmland soils by toxic and/or hazardous organic pollutants is a global environmental problem. ${ }^{1}$ The long-term utilization of agricultural pesticides and repeated fertilization with animal manure contaminated with antibiotics have resulted in widespread and co-existing contaminations in agricultural soils. ${ }^{2,3}$ Such contaminations pose a potential environmental risk to humans and microorganisms through environmental exposure and migration., ${ }^{\mathbf{4} 5}$ Sorption and immobilization are two critical processes that govern the mobility, bioavailability, and the ultimate fate of the contaminants in soils. ${ }^{6}$ Therefore, the possibility of exploiting in situ application of amendments capable of binding pesticides and antibiotics in contaminated soils, thereby reducing their environmental risk, has received great attention recently.,

\footnotetext{
${ }^{a}$ Department of Environmental Science, Zhejiang University, Hangzhou, Zhejiang 310058, China. E-mail: wenjunzhou@zju.edu.cn; Fax: +86-571-88982591; Tel: +86571-88982591

${ }^{b}$ Zhejiang Provincial Key Laboratory of Organic Pollution Process and Control, Hangzhou, Zhejiang 310058, China

${ }^{c}$ Center of Analysis and Measurement, Zhejiang University, Hangzhou 310058, China

$\dagger$ Electronic supplementary information (ESI) available. See DOI: 10.1039/c6ra26534k
}

Biochars are carbonaceous materials produced by the pyrolysis of biomass residues from agricultural and forestry production under oxygen-deficient condition. ${ }^{9}$ Increasingly, these materials are receiving recognition as potential amendments that could help reduce the migration and bioavailability of organic contaminants in soils, thereby reducing their environmental risk. ${ }^{9,10}$

Owing to their large specific surface area (SSA), and high hydrophobicity and aromaticity, biochars exhibit an extraordinarily strong adsorption affinity for a number of hydrophobic organic contaminants (HOCs). ${ }^{\mathbf{1 1 , 1 2}}$ A better understanding of the sorption characteristics and mechanisms of pesticide and antibiotic onto biochars is essential for effective implementation of biochars as amendments to agricultural soils.

To date, much work has been conducted to investigate the sorption of organic contaminants by biochars. Several recent studies have revealed that the sorption affinity and maximum capacity of biochars for pesticides and antibiotics depended on their degree of carbonization, and surface and bulk properties such as the pore size distribution, specific surface area, hydrophobicity, and polarity. ${ }^{6,11,13,14}$ In addition, the physicochemical properties of the sorbates (e.g., the molecular weight, hydrophobicity, and polarity) and the solution chemistry conditions (e.g., $\mathrm{pH}$ and ionic strength) were also found to play a key role in determining the sorption mechanisms of pesticides and antibiotics by 
biochars. ${ }^{15-17}$ Most of these studies have focused on examining the source material from which the biochar was produced and the thermochemical conditions employed in the biochar pyrolysis, ${ }^{6,18,19}$ as these parameters are known to affect the specific surface area, microporosity, and surface chemistry of biochars. To date, however, investigations directed at examining the impact of the biochar mineral component and its interactions with organic matters of biochars are rather limited in the literature. ${ }^{20-25}$

The published studies examining the impact of the mineral content of natural soils and carbonaceous materials on the sorption of organic pollutants have focused mainly on single-solute sorption system. ${ }^{6,15,20,21,23-27}$ The results of these studies have shown that the mineral components and their interactions with the organic matter present in carbonaceous materials exerted a great influence on their overall sorption capacity. On one hand, mineral components can inhibit the non-specific adsorption of organic molecules by blocking access to the pores of carbonaceous materials. ${ }^{15,21,25,28}$ On the other hand, mineral surfaces can enhance the sorption of polar organic chemicals through site-specific interactions such as electron donor-acceptor and hydrogen-bonding interactions. ${ }^{22,25,29,30}$ For ionic organic compounds, minerals may provide sites for cation or anion binding interaction. ${ }^{16}$ Therefore, it seems reasonable to hypothesize that polar pesticide and ionic antibiotic could be capable of forming strong complexes with (i) the hydroxyl groups of inorganic silicates and other minerals, and (ii) the electron-rich aromatic structures of organic components (e.g., graphite surfaces) associated with biochars. Combined together, the mineral components of biochars and their interactions with organic moieties are expected to influence the overall sorption characteristics of pesticides and antibiotics on biochars. However, studies examining the impact of mineral content of biochars on the overall adsorption and competitive adsorption of co-existing pesticides and antibiotics are extremely scarce.

Biochars derived from livestock manure usually possess a high mineral content, ${ }^{6,15,19}$ for this reason, we employed cow manure biochars as the adsorbents in this study. A carbamate insecticide (carbaryl) and a tetracycline antibiotic (oxytetracycline) were selected as a model pesticide and antibiotic, respectively. These compounds have been selected in particular because they have been widely detected in the surface water and in agricultural soil. ${ }^{31,32}$ The adsorption of carbaryl and oxytetracycline on original and demineralized biochars in single- and binary-solute systems were investigated and the main adsorption mechanisms were discussed. The objectives of this study are to evaluate the impact of mineral components in biochars on the adsorption and competitive adsorption of a pesticide and an antibiotic, and thus develop a better understanding of their sorption mechanisms. The obtained results are expected to provide a firm theoretical groundwork for further analyses of the potential application of biochars in the remediation of combined pollution in agricultural soils.

\section{Materials and methods}

\subsection{Materials}

Carbaryl (CBL) was obtained from Sigma-Aldrich (USA) with a reported purity of $97 \%$. Oxytetracycline hydrochloride (OTC) was supplied by J\&K Chemical Co. Ltd. (Beijing, China) with a reported purity of $95 \%$. Other chemicals used in this study were purchased from Aladdin Chemistry Co. Ltd. (China) (analytical or HPLC grade). The molecular structure and selected physicochemical properties of OTC and CBL are presented in Table 1.

Cow manure stock was collected from a hoggery in Longyou County, Zhejiang, China. The samples were air-dried, ground, passed through a $2 \mathrm{~mm}$ sieve, and pyrolyzed in a preheated muffle furnace at either $300,400,500,600$, or $700{ }^{\circ} \mathrm{C}$ for $6 \mathrm{~h}$ under oxygen-deficient conditions as described by $\mathrm{Zhu}$ et al. $^{\mathbf{1 1}}$ The prepared biochars were referred to as CMBCX, where CMBC referred to the cow manure-derived biochar, and $X$ referred to the pyrolysis temperature. The produced biochars were ground and passed through 200-mesh sieve and stored in amber glass bottles at room temperature for later use.

Demineralized biochars were prepared using the method described by Yang et al. $^{33}$ for elimination of inorganic components. Briefly, the original biochars CMBC600 and CMBC700 were first treated by repeated rinsing with aqueous $\mathrm{HCl}(1 \mathrm{M})$, followed by rinsing with a mixture of aqueous $\mathrm{HCl}(1 \mathrm{M})$ and $\mathrm{HF}$ (1 M), and lastly, by rinsing with distilled water several times until solution $\mathrm{pH}$ was neutral, in order to remove any residual acid and soluble salts. The resulting products were freeze-dried and stored in desiccators for later use. Using the original notation based on the pyrolysis temperature, these demineralized biochar samples were referred to as DCMBC600 and DCMBC700, where D denotes demineralized. The prepared biochars were characterized by bulk elemental compositions analysis and surface elemental compositions analysis using an elemental analyzer (Thermo Finnigan Flash EA 1112), and X-ray photoelectron spectroscopy (XPS) (ULVAC-PHI PHI 5000 VersaProbeII, Japan). The ash content of biochars was measured by determining the residual weight after the biochars were heated at $900{ }^{\circ} \mathrm{C}$ for $4 \mathrm{~h}$. The oxygen content was calculated by mass difference. The surface area, pore volume, and identity of surface functional groups were characterized using the Brunauer-Emmett-Teller (BET) analysis, and Fourier-transform infrared spectroscopy (FTIR).

\subsection{Batch sorption experiments}

Batch experiments were conducted to determine the sorption of OTC and CBL onto the original (CMBCs) and demineralized biochars (DCMBCs) in triplicate in single- or binary-solute systems. These experiments were performed in $22 \mathrm{~mL}$ Corex centrifuge tubes equipped with Teflon-lined screw caps, in which an appropriate amount of the biochar was mixed with 20 $\mathrm{mL}$ of aqueous solutions containing OTC and/or CBL. The background solution $(\mathrm{pH}=5.5)$ contained $\mathrm{CaCl}_{2}(0.01 \mathrm{M})$ and $\mathrm{NaN}_{3}\left(200 \mathrm{mg} \mathrm{\textrm {L } ^ { - 1 }}\right)$ in order to maintain a constant ionic strength and to inhibit microbial activity, respectively. On the basis of preliminary tests, the samples were equilibrated on a reciprocating shaker $(160 \mathrm{rpm})$ for $24 \mathrm{~h}$ at $25 \pm 1{ }^{\circ} \mathrm{C}$, and then centrifuged at $3000 \mathrm{rpm}$ for $6 \mathrm{~min}$. An appropriate aliquot of the supernatant was filtered through a $0.45 \mu \mathrm{m}$ glass fiber membrane into $2 \mathrm{~mL}$ glass vials for the analysis of the OTC and 
Table 1 Molecular structure and selected physicochemical properties of oxytetracycline (OTC) and carbaryl (CBL)

Compound

Structure and size ${ }^{a}$
OTC

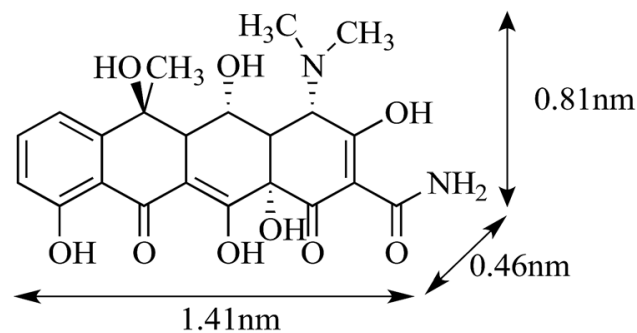

$\mathrm{C}_{22} \mathrm{H}_{24} \mathrm{~N}_{2} \mathrm{O}_{9}$

460.43

-

0.11-0.34

$3.27,7.32,9.11$
CBL

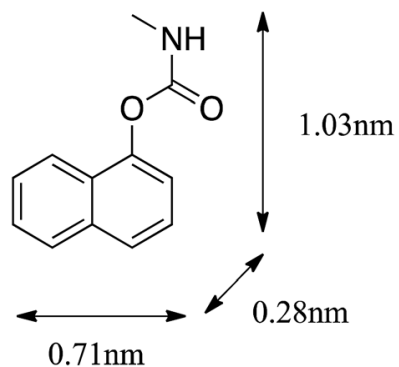

$\mathrm{C}_{12} \mathrm{H}_{11} \mathrm{NO}_{2}$

201.22

82.6

2.36

$K_{\text {ow }}{ }^{e}$

${ }^{a}$ Values obtained from Ji et al. ${ }^{20}$ and Zhang et al. ${ }^{15 b} \mathrm{MW}$, molecular weight. ${ }^{c} S_{\mathrm{w}}$, solubility in water (in mg L ${ }^{-1}$ ), data from Marie et al. ${ }^{39}{ }^{d} K_{\mathrm{ow}}$, octanol-water partition coefficient, data from Kulshrestha et al. ${ }^{29}$ and USEPA. ${ }^{40} e{ }_{\mathrm{a}}$, acid dissociation constant, data from Kulshrestha et al. ${ }^{29}$

CBL content by HPLC. The concentration of the adsorbed solute was calculated simply by taking the difference between the initial and the equilibrium solute concentrations in the aqueous phase.

In single-solute sorption experiments, the initial aqueous concentrations of OTC and CBL were $4-40 \mathrm{mg} \mathrm{L}^{-1}$ and $8-80 \mathrm{mg}$ $\mathrm{L}^{-1}$, respectively. Binary-solute sorption experiments were conducted on CMBC600 and $\mathrm{CMBC700,} \mathrm{and} \mathrm{the} \mathrm{corresponding}$ demineralized biochars DCMBC600 and DCMBC700. In these experiments, OTC or CBL was selected as the primary sorbate (4-40 $\mathrm{mg} \mathrm{L}^{-1}$ concentration range), with CBL or OTC as the cosorbate at a fixed concentration of $32 \mathrm{mg} \mathrm{L}^{-1}$.

\subsection{Quantification and data analysis}

The concentrations of OTC and CBL in the aqueous solution were quantified using an Agilent 1100 HPLC (USA) fitted with a UV detector and an Agilent Eclipse XDB-C18 column $(4.5 \times 15$ $\mathrm{mm}, 5 \mu \mathrm{m})$. Acetonitrile/oxalic acid (0.01 M) (20:80, v/v) and acetonitrile/oxalic acid (0.01 M) (65:35, v/v) at a flow rate of 1.0 $\mathrm{mL} \min ^{-1}$ were employed as the mobile phase for OTC and CBL, respectively. The UV wavelengths were set at $360 \mathrm{~nm}$ for OTC and $254 \mathrm{~nm}$ for CBL, respectively.

The isotherm data obtained in this study were fitted to the Freundlich model adsorption as described by eqn (1) or (2):

$$
Q_{\mathrm{e}}=K_{\mathrm{F}} C_{\mathrm{e}}^{n}
$$

or

$$
\log Q_{\mathrm{e}}=\log K_{\mathrm{F}}+n \log C_{\mathrm{e}},
$$

where $Q_{\mathrm{e}}\left(\mathrm{mg} \mathrm{kg}^{-1}\right)$ and $C_{\mathrm{e}}\left(\mathrm{mg} \mathrm{L}^{-1}\right)$ represent the equilibrium solid and aqueous concentrations, respectively. $K_{\mathrm{F}}\left[\mathrm{in}\left(\mathrm{mg} \mathrm{kg}^{-1}\right)\right.$ $\left(\mathrm{mg} \mathrm{L}^{-1}\right)^{-n}$ ] and $n$ denote the sorption affinity and the nonlinearity index, respectively.

\section{Results and discussion}

\subsection{Characterization of biochars}

The bulk elemental compositions, ash content, atomic ratios, surface area and pore volume parameters of the biochars tested in this study are shown in Table 2. The mineral content of original CMBCs was found to be (i) significantly higher than that of biochars derived from plant sources ${ }^{8}$ and (ii) to increase with the increasing pyrolysis temperature. The atomic ratios of $\mathrm{H} / \mathrm{C}, \mathrm{O} / \mathrm{C}$, and $[(\mathrm{O}+\mathrm{N}) / \mathrm{C}]$ are generally recognized as indexes for aromaticity, hydrophobicity, and polarity, respectively. ${ }^{\mathbf{1 3 , 1 4}}$ These three atomic ratios determined for the original biochars were found to decrease with the increasing pyrolysis temperature, indicating an increase in the bulk aromaticity and hydrophobicity, but a decrease in the bulk polarity. These results are also supported by the acquired FTIR spectra (Fig. S1 $\dagger$ ). Briefly, the transformation of the aliphatic carbons into aromatic carbons can be evidenced by the reduction in the intensity of the peaks at 2993 and $1489 \mathrm{~cm}^{-1}$ and the enhancement in the intensities of the bands at 849, 709, and $638 \mathrm{~cm}^{-1} \cdot{ }^{34}$ The diminished intensity of the bands at $3690 \mathrm{~cm}^{-1}$ and 1783-1106 $\mathrm{cm}^{-1}$ indicates a reduction in polarity.

The demineralization treatment of CMBC600 and CMBC700 resulted in essentially complete removal of the mineral components (Table 2). The surface elemental compositions as determined by XPS and surface atomic ratios of the prepared biochars are presented in Table S1. $\dagger$ Carbon was found to be the dominant surface element, followed by oxygen. The main mineral element present on the surface of these biochars was Si. The content of most surface elements decreased, with the exception of $\mathrm{C}$ and $\mathrm{N}$, while most mineral elements could even not be detected at all. The surface $\mathrm{C}$ content of original CMBCs was obviously higher than their corresponding bulk $\mathrm{C}$, suggesting the minerals of these original biochars were likely covered by organic component as well as the structural heterogeneity within biochars as reported by Zhang et al. ${ }^{15}$ and 
Table 2 Bulk elemental composition, atomic ratios, surface area, and pore volume parameters of original (CMBCs) and demineralized (DCMBCs) biochars

Bulk elemental composition $^{a}(\%)$

Atomic ratio $^{c}$

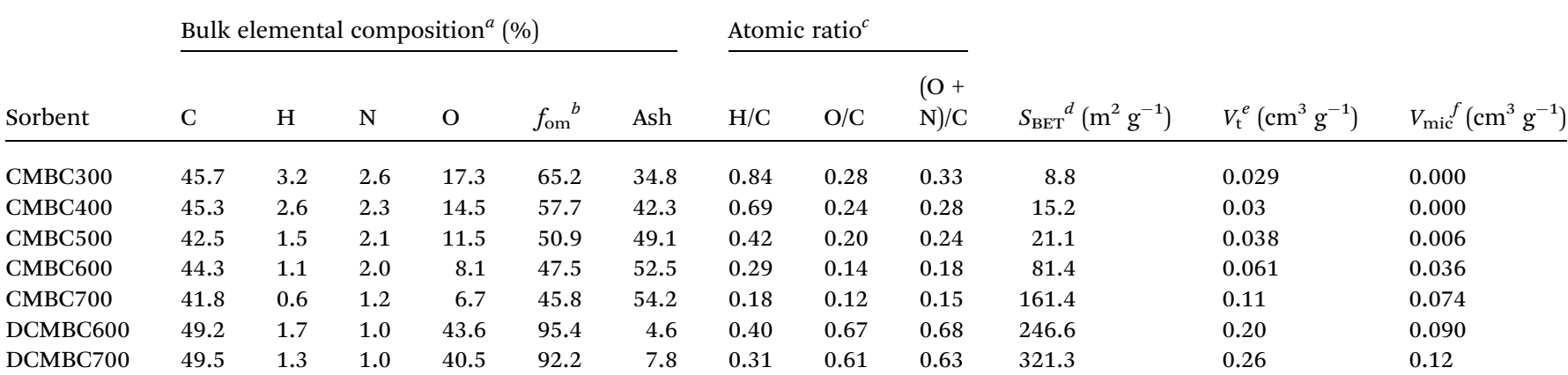

${ }^{a}$ Determined using an elemental analyzer. ${ }^{b}$ Organic matter content. ${ }^{c}$ Calculated from the atomic ratio of the bulk elements. ${ }^{d}$ Determined by $\mathrm{N}_{2}$ adsorption using the Brunauer-Emmett-Teller (BET) method. ${ }^{e}$ Total pore volume, determined at $P / P_{0}=0.99 .{ }^{f}$ Micropore volume, calculated using the $t$-plot method. ${ }^{41}$

Sun et al. ${ }^{21}$ Furthermore, the increased surface $\mathrm{C}$ contents of the CMBCs after demineralization was consistent with their increased bulk $\mathrm{C}$ amounts (Tables 2 and S1†), suggesting that minerals of these CMBCs had no obvious effect on the spatial distribution of organic matter, which is not consisted with the result reported by Sun et al. ${ }^{21}$

The demineralization treatment also decreased the bulk aromaticity and hydrophobicity, while at the same time increased the bulk polarity of the original biochars, as indicated by the increasing atomic ratios of $\mathrm{H} / \mathrm{C}, \mathrm{O} / \mathrm{C}$, and $[(\mathrm{O}+\mathrm{N}) / \mathrm{C}]$ in the demineralized biochars (Table 2). The FTIR spectra can also verify the result mentioned above (Fig. S1 $\dagger$ ). However, the surface hydrophobicity of original biochars was found to increase while the surface polarity decreased after demineralization treatment (Table $\mathrm{S} 1 \dagger$ ), which was a trend opposite to that observed for the bulk properties. This difference in properties suggests that abundant minerals of biochars would benefit the exposure of polar groups on the surface of the tested biochars, which could exhibit higher surface polarity of original biochars than their corresponding demineralized ones. ${ }^{21}$

The surface area and the pore volume of all biochars were found to increase with increasing pyrolysis temperature, and a particularly large difference was observed for the biochars produced at the highest temperature (Table 2). For CMBC600 and $\mathrm{CMBC} 700$, the micropore volumes accounted for a significant proportion (58-69\%) of the total pore volumes (Fig. S2 $\dagger$ ). After demineralization, the surface area and the micropore volume of biochars increased much more significantly, with the greatest change observed for DCMBC700. However, the percentage of the micropore volume in the total pore volume decreased to $44-47 \%$ for DCMBC600-DCMBC700 because of the increase in their mesopore volume.

\subsection{Sorption isotherms of oxytetracycline and carbaryl on original cow manure biochars}

The sorption isotherms and Freundlich model fitting parameters determined for the adsorption of oxytetracycline and carbaryl on five original CMBCs are shown in Fig. 1 and Table $\mathrm{S} 2, \uparrow$ respectively. The sorption isotherms of oxytetracycline and carbaryl on original CMBCs were nonlinear and the sorption datas were fitted successfully to the Freundlich model $\left(R^{2}>0.95\right.$ in each case). The sorption affinities of the original biochars for both oxytetracycline and carbaryl increased with increasing pyrolysis temperature. This increase was found to be especially noticeable for CMBC600 and CMBC700. The corresponding adsorption affinity $\left(K_{\mathrm{F}}\right)$ for OTC and CBL increased from $10^{3.05}$ to $10^{3.77}$ and from $10^{3.32}$ to $10^{4.43}$, respectively, which are
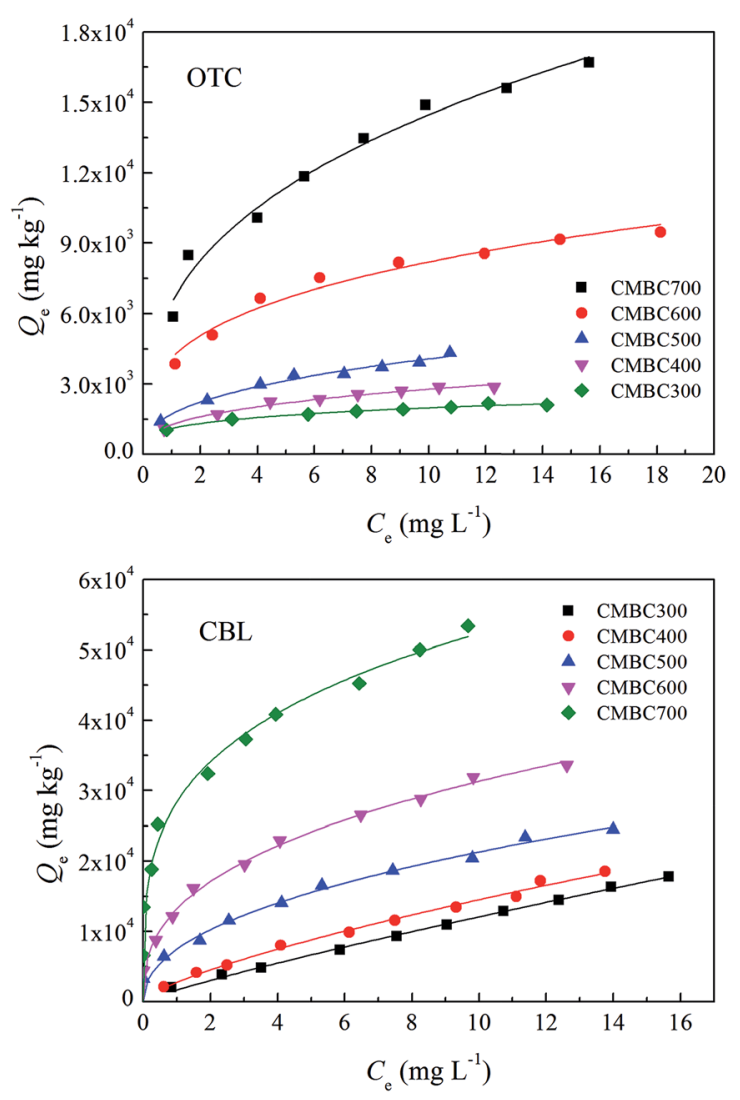

Fig. 1 Adsorption isotherms of oxytetracycline (OTC) and carbaryl (CBL) on original biochars pyrolyzed at different temperatures (300$\left.700{ }^{\circ} \mathrm{C}\right)$. 
markedly higher than the values reported in other studies for OTC $\left(10^{2.58}\right.$ to $\left.10^{2.9}\right)$ and CBL $\left(10^{2.9}\right.$ to $\left.10^{3.74}\right)$ on biochars. ${ }^{8,17}$ In addition, the sorption amount and the sorption affinity $K_{\mathrm{F}}$ of CMBCs for CBL were found to be higher than those for OTC. The nonlinearity of the CBL sorption isotherms increased with increasing pyrolysis temperature, and the values of $n$ decreased from 0.762 to 0.282 . Moreover, the values of $n$ in the OTC sorption isotherms were much lower than those obtained for CBL on CMBCs, exhibiting a slight increase from 0.247 to 0.395 (except CMBC600). The lower value of $n$ for the biochars indicates a higher number of heterogeneous glass, hard or condensed sorption domains, and a larger sorption site energy distribution. ${ }^{35}$ These results are indicative of a better distribution of high-energy adsorption sites for OTC than for CBL on the original biochars.

According to the results presented above, CMBCs derived at different temperatures displayed dissimilar sorption characteristics for OTC and CBL. To further elucidate the factors that govern the adsorption of OTC and CBL on CMBCs, the chemical structural parameters of these biochars were correlated with the organic carbon-normalized sorption coefficient $\left(\log K_{\mathrm{oc}}\right.$ values at three different sorbate concentrations, Table S2 $\dagger$ ) and the nonlinearity index ( $n$ values, Table S2 $\dagger$ ) of OTC and CBL (Fig. S3 and $\mathrm{S} 4 \dagger)$. This comparison showed that the $\log K_{\mathrm{oc}}$ values of OTC and CBL at all three different sorbate concentrations were significantly negatively correlated with the $\mathrm{H} / \mathrm{C}, \mathrm{O} / \mathrm{C}$, and $[(\mathrm{O}+$ $\mathrm{N}) / \mathrm{C}$ ] atomic ratios of the original biochars, revealing the positive effect of the aromatic and hydrophobic carbon contents of biochars and the negative effect of their bulk polarity on the OTC and CBL adsorption. These observations are in agreement with the findings presented in recent studies, ${ }^{15,20,36}$ which have shown that OTC and CBL can interact strongly with the aromatic surfaces of carbonaceous adsorbents via strong $\pi-\pi$ electron donor-acceptor (EDA) interactions. Additionally, it has been well-documented in literatures ${ }^{11,37}$ that the adsorption of HOCs to carbonaceous adsorbents can be inhibited by surface polar oxygen-containing functional groups because these groups can facilitate formation of larger and denser water molecule clusters around them, and in turn, creates a stronger competition for the surface area available on the graphitized carbon of adsorbents. Hence, in the present study, the decrease in the $\mathrm{H} / \mathrm{C}$ and $\mathrm{O} / \mathrm{C}$ atomic ratios of the original biochars is expected to enhance the $\pi-\pi$ EDA interactions, contributing to the sorption of OTC and CBL. Additionally, the aromaticity of CMBCs in this study was higher than the aromaticity of biochars examined in the prior sorption study for OTC and CBL, ${ }^{\mathbf{8 , 1 7}}$ and thus, the CMBCs showed a higher sorption affinity for both OTC and CBL.

Fig. $\mathrm{S} 4 \dagger$ clearly shows that the values of $n$ in the CBL sorption isotherms on the original CMBCs were significantly positively correlated with the $\mathrm{H} / \mathrm{C},[(\mathrm{O}+\mathrm{N}) / \mathrm{C}]$, and $\mathrm{O} / \mathrm{C}$ atomic ratios, but no significant correlation was found between the $n$ values in the OTC sorption isotherms and the chemical structure parameters of the original biochars. Therefore, the increase in the aromatic and hydrophobic organic components in the CMBCs with an increasing pyrolysis temperature is the major factor contributing to the nonlinearity of the CBL sorption isotherms, which can provide specific sorption sites for $\pi-\pi$ EDA interactions and hydrophobic effect. Although no significant correlation was found between the values of $n$ for OTC and the chemical structural properties of the original biochars, the $n$ values of OTC were markedly lower than those of CBL. This difference suggests that pore-filling at the limited surface and/or interior sites may be the dominant mechanism for OTC adsorption. ${ }^{35}$ In comparison to CBL, OTC is a much larger molecule $(1.41 \mathrm{~nm}$ versus $0.71 \mathrm{~nm}$, see Table 1), and it may, therefore, experience pronounced steric hindrance when approaching the sorption domains in the micropores, resulting in a weaker sorption affinity than that of CBL.

\subsection{Impact of mineral components on the adsorption of oxytetracycline and carbaryl on biochars}

As evidenced by the characterization analyses of biochars, the demineralization treatment has significantly increased the surface area and the micropore volume of original biochars. The treatment also altered their chemical characteristics, which may manifest in completely different sorption characteristics of the demineralized biochars when compared to the original biochars. To test this hypothesis, the sorption isotherms of OTC and CBL on the original biochars and the corresponding demineralized biochars are compared in Fig. 2, and the Freundlich model parameters are listed in Table 3. It is observed that the sorption isotherms of OTC and CBL on the demineralized biochars also exhibit nonlinearity, and the corresponding values of $n$ decreased drastically after the demineralization treatment. Therefore, the demineralization treatment increased the degree of nonlinearity of the OTC and CBL sorption isotherms on biochars. However, the demineralization showed different effect on the sorption affinity of OTC and CBL on the biochars. It can be clearly seen from Fig. 2 that the OTC uptake onto the selected biochars decreased in the following: DCMBC700 > DCMBC600 > CMBC700 > CMBC600, and for CBL uptake: CMBC700 > DCMBC700 > CMBC600 > DCMBC600. These trends show that the demineralization treatment increased significantly the adsorption of OTC on biochars, but decreased slightly the adsorption of CBL.

The demineralization treatment removed the majority of the mineral components and created a large number of additional micropores on the biochars, which possess a wide distribution of sorption site energies and can increase the degree of isotherm nonlinearity. ${ }^{21,35}$ Therefore, demineralization may enhance the site-specific interactions and pore-filling on the biochars and give rise to the significantly nonlinear adsorption of OTC and CBL. This sorption enhancement, however, could be somewhat counteracted by the contributions of the mineral components-it is well documented in the literature that tetracyclines can adsorb strongly on the surfaces of iron/ aluminum hydroxides and clay minerals via surface complexation (e.g., mediated by $\mathrm{H}$-bonding) and/or ion exchange reactions. ${ }^{29,30}$ Furthermore, studies have shown that clay minerals can adsorb CBL through site-specific interactions between the carbamate functional groups and the exchangeable cations present on the mineral surface. ${ }^{26}$ 

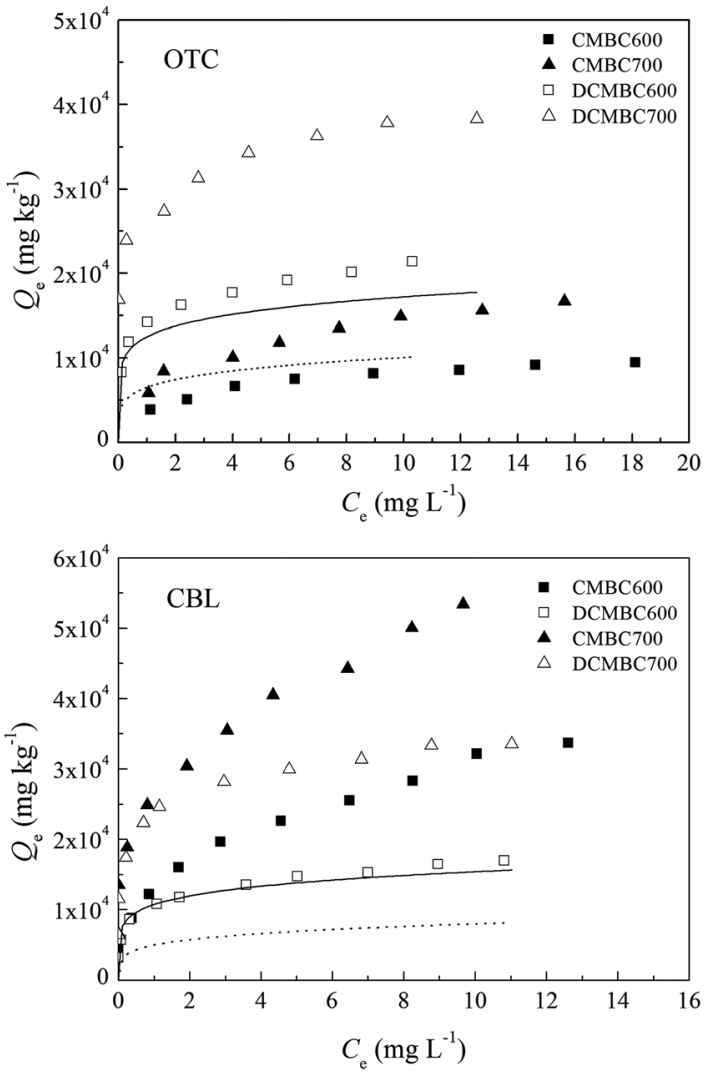

Fig. 2 Comparison of the adsorption isotherms of oxytetracycline (OTC) and carbaryl (CBL) on original (CMBCs) and demineralized (DCMBCs) biochars. The solid and dotted lines represent the estimated Freundlich adsorption isotherms for CMBC700 and CMBC600, respectively.
In order to determine the impact of the mineral components in the original biochars on its overall adsorption of OTC and $\mathrm{CBL}$, the measured adsorption isotherms for the demineralized biochars were fractionalized according to the content of organic matter of the original biochars. The corresponding Freundlich fitting curves (estimated isotherms) are also shown in Fig. 2, representing the adsorption capacity of the organic matter fraction in the original biochars. The differences between the measured and the estimated sorption isotherms represent the minimum adsorption contribution or adsorption inhibition of the mineral fraction. For OTC, the estimated isotherms were located slightly above the measured isotherms, with differences of less than 25\% for CMBC700, and less than 15\% for CMBC600. However, these trends were completely reversed for CBL, as showed in Fig. 2. Namely, the estimated CBL isotherms were placed well below the measured isotherms for both CMBC600 and $\mathrm{CMBC} 700$, with a difference of more than $50 \%$ in each case. Based on the comparison of the estimated and the measured sorption isotherms for OTC and CBL, it can be established that the mineral components inhibit slightly the overall sorption of OTC to the original biochars, and enhance significantly the sorption of CBL. However, this observation is not consistent with previously reported studies, ${ }^{15,20}$ in which the mineral content was found to have a negative influence on the overall adsorption of CBL to biochars, and a significant positive contribution for tetracycline adsorption to black carbon. This discrepancy could be attributed to the differences in the main mechanisms of adsorption of OTC and CBL on the demineralized biochars.

The demineralization treatment decreased the bulk aromaticity and hydrophobicity of biochars but increased their bulk polarity, while the $\log K_{\mathrm{oc}}$ of OTC still increased significantly, which means that it is not the site-specific sorption mechanism

Table 3 Freundlich model parameters and concentration-dependent distribution coefficients $\left(K_{\text {oc }}\right)$ for oxytetracycline (OTC) and carbaryl (CBL) adsorption/competition isotherms on selected original (CMBCs) and demineralized (DCMBCs) biochars

\begin{tabular}{|c|c|c|c|c|c|c|c|c|}
\hline & \multirow[b]{2}{*}{ Sorbent } & \multirow[b]{2}{*}{ Competitor } & \multirow[b]{2}{*}{$\log K_{\mathrm{F}}$} & \multirow[b]{2}{*}{$n$} & \multirow[b]{2}{*}{$R^{2}$} & \multicolumn{3}{|c|}{$\underline{\log K_{\mathrm{oc}}{ }^{a} \text { at }}$} \\
\hline & & & & & & $C_{\mathrm{e} 1}^{b}$ & $C_{\mathrm{e} 2}{ }^{b}$ & $C_{\mathrm{e} 3}^{b}$ \\
\hline & & $32 \mathrm{mg} \mathrm{L}^{-1} \mathrm{CBL}$ & 3.48 & 0.364 & 0.964 & 4.361 & 3.725 & 3.089 \\
\hline & \multirow[t]{2}{*}{ CMBC700 } & - & 3.77 & 0.395 & 0.978 & 4.646 & 4.041 & 3.436 \\
\hline & & $32 \mathrm{mg} \mathrm{L}^{-1} \mathrm{CBL}$ & 3.76 & 0.428 & 0.923 & 4.683 & 4.011 & 3.339 \\
\hline & \multirow[t]{2}{*}{ DCMBC700 } & - & 4.42 & 0.164 & 0.983 & 5.309 & 4.473 & 3.637 \\
\hline & & $32 \mathrm{mg} \mathrm{L}^{-1} \mathrm{CBL}$ & 4.25 & 0.254 & 0.981 & 5.076 & 4.330 & 3.584 \\
\hline \multirow{4}{*}{ CBL } & \multirow[t]{2}{*}{ СMBC600 } & - & 4.11 & 0.382 & 0.973 & 4.783 & 4.165 & 3.547 \\
\hline & & $32 \mathrm{mg} \mathrm{L}^{-1}$ OTC & 3.60 & 0.845 & 0.995 & 4.096 & 3.941 & 3.786 \\
\hline & \multirow{2}{*}{ CMBC700 } & - & 4.43 & 0.282 & 0.887 & 5.159 & 4.441 & 3.723 \\
\hline & & $32 \mathrm{mg} \mathrm{L}^{-1}$ OTC & 3.98 & 0.667 & 0.976 & 4.561 & 4.228 & 3.895 \\
\hline
\end{tabular}

${ }^{a} K_{\mathrm{oc}}$ is the concentration-dependent organic carbon-normalized sorption distribution coefficient $\left(K_{\mathrm{oc}}=K_{\mathrm{F}} C_{\mathrm{e}}{ }^{n-1} / f_{\mathrm{oc}}\right)$, in L kg ${ }^{-1}$ of organic carbon, where $f_{\mathrm{oc}}$ is the percentage of carbon content of biochar. ${ }^{b}$ For OTC, $C_{\mathrm{e} 1}, C_{\mathrm{e} 2}$, and $C_{\mathrm{e} 3}$ were $0.2,2.0$, and $20 \mathrm{mg} \mathrm{L}^{-1}$, respectively. For CBL, $C_{\mathrm{e} 1}, C_{\mathrm{e} 2}$, and $C_{\mathrm{e} 3}$ equaled to $0.005 S_{\mathrm{w}}, 0.05 S_{\mathrm{w}}$, and $0.5 S_{\mathrm{w}}$, respectively. $S_{\mathrm{w}}\left(\mathrm{mg} \mathrm{L}^{-1}\right)$ is the solubility of carbaryl in water under neutral pH. 
but another sorption mechanism that contributed to the OTC sorption the most. The demineralization process removed most of the mineral content of CMBC600 and CMBC700, thereby reducing the contribution of the mineral components to the adsorption of OTC. However, the demineralization simultaneously released a large number of additional surface micropore sorption sites as pores and surface areas drastically increased, thereby enhancing the accessibility of the sorption sites and pore-filling effect for the adsorption of OTC. Namely, the increase in the $\log K_{\mathrm{oc}}$ values and the decrease in the values of $n$ (well lower than 1) for the OTC sorption isotherms on the demineralized biochars, together with the increased surface area and the number of pores are indicative of the pore-filling mechanism. In addition, the surface oxygen content of the demineralized biochars was found to be notably lower than that of the original biochars (see Table S1 $\dagger$ ). Therefore, the demineralization treatment would be expected to reduce water molecule clusters competition adsorption mentioned above. Hydrogen-bonding interactions between the OTC and the biochars might also be enhanced ${ }^{6}$ following demineralization because the content of certain polar groups increased, as evidenced by the changes in the $[\mathrm{O}+\mathrm{N}] / \mathrm{C}$ atomic ratio and the results of the FTIR spectroscopy analysis. The sorption enhancements mentioned above could counterbalance the loss of mineral contents sorption induced by demineralization, and potentially even can increase the overall adsorption of OTC. Nevertheless, while the number of micropores and the surface area increased significantly after demineralization, the $\pi-\pi$ EDA and ion exchange interactions between CBL and biochars may be reduced as a result of the decrease in the aromatic and mineral content, thus actually leading to an overall decrease in adsorption. Hence, it is hypothesized that the main mechanism of OTC sorption on the demineralized biochars is the porefilling effect, whereas the CBL adsorption can be attributed to the specific site adsorption such as $\pi-\pi$ EDA interactions.

Fig. S2 $\uparrow$ shows the results of the pore size distribution analysis. While the demineralized biochars were found to be highly microporous, the ratio of micropore volume to the total pore volume was lower compared to that of original biochars. This difference can be attributed to the increase in the mesopore volume following demineralization. Meanwhile, the two examined sorbates vary significantly in molecular size, and thus, may exhibit size-dependent adsorption on the selected biochars, regulated by the porosity of the adsorbent. To test this hypothesis, the adsorbent surface area-normalized adsorption isotherms of OTC and CBL on two original and two demineralized biochars are shown in Fig. 3. Comparison of the original sorption isotherms (Fig. 2) and the adsorbent surface areanormalized adsorption isotherms (Fig. 3) reveals that the differences in the adsorption affinities between the original and demineralized biochars for OTC are significantly larger in the original sorption isotherms than in the surface areanormalization sorption isotherms. This outcome indicates that the higher adsorption affinity of the demineralized biochars for OTC than that of the original ones can be largely attributed to their larger surface area and mesopore volume, and further confirms the pore-filling effect as the main sorption
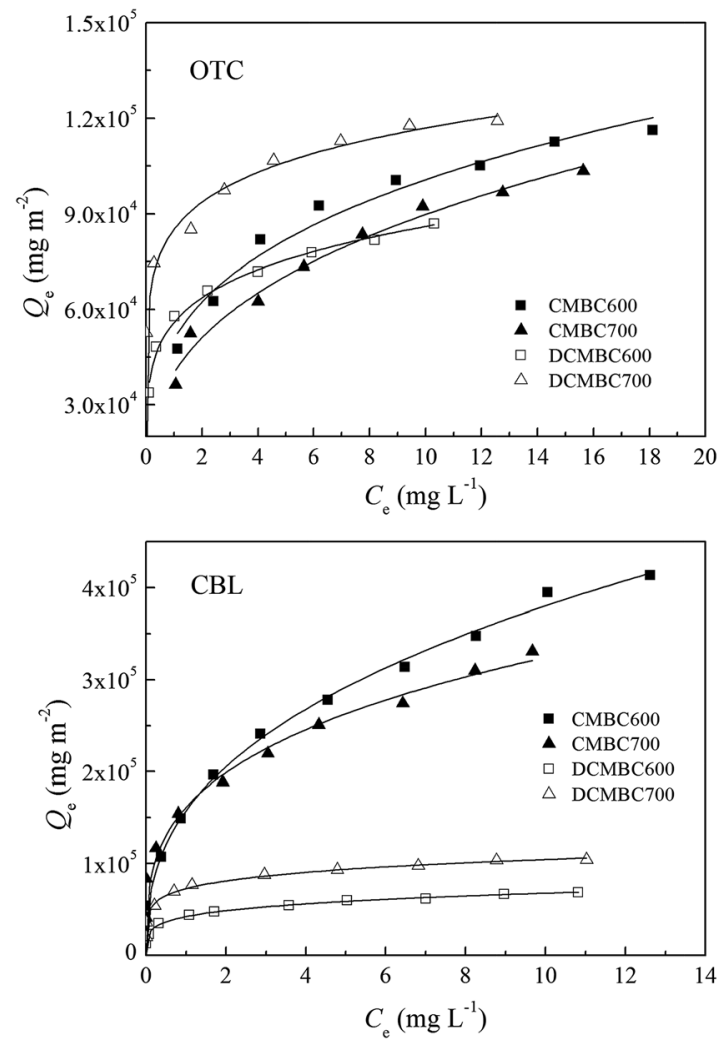

Fig. 3 Adsorbent surface area-normalized adsorption isotherms for oxytetracycline (OTC) and carbaryl (CBL) on original (CMBCs) and demineralized (DCMBCs) biochars.

mechanism for OTC on demineralized biochars. In contrast, the differences in the adsorption affinities for CBL between the original and demineralized biochars are much more noticeable in the surface area-normalized isotherms than that in the original isotherms. This means that it is not the increase in the biochar surface area that plays a key role in the decrease of the adsorption affinity for CBL but other factors induced by the demineralization process. These results have demonstrated that the sorption of OTC and CBL on demineralized biochars proceeds through different mechanisms.

\subsection{Competitive adsorption of oxytetracycline and carbaryl on original and demineralized biochars}

The competitive sorption between different chemicals generally occurs at the solid-water interface, and can affect the sorption characteristic and affinity of the other contaminant on the adsorbent. Therefore, the adsorption of oxytetracycline and carbaryl on the original and demineralized biochars in binarysolute system was investigated. The sorption isotherms for OTC and CBL on selected CMBCs and corresponding DCMBCs with and without a co-solute are shown in Fig. 4, and their Freundlich fitting parameters are listed in Table 3 . The presence of OTC was found to significantly inhibit the sorption of CBL on the original biochars (CMBC600 and $\mathrm{CMBC} 700$ ), indicating a competitive effect of OTC on CBL sorption. However, no significant changes were observed in the sorption of OTC 

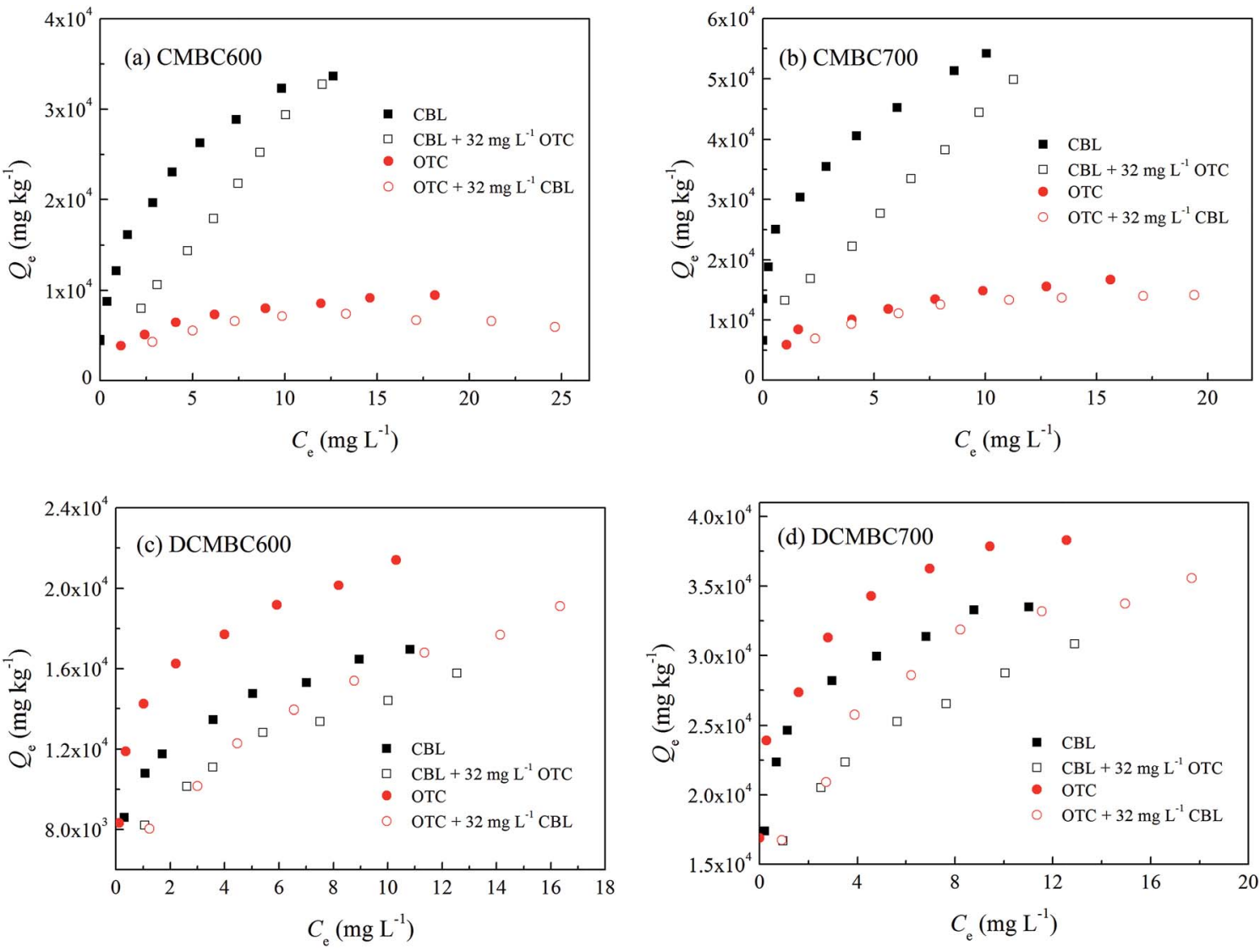

Fig. 4 Adsorption isotherms of carbaryl (CBL) and oxytetracycline (OTC) on original (CMBCs) and demineralized (DCMBCs) biochars with and without a co-solute ([OTC] or [CBL] $\left.=32 \mathrm{mg} \mathrm{L}^{-1}\right)$.

following the addition of CBL (Fig. $4 \mathrm{a}$ and b), suggesting that the CBL displayed negligible competition with OTC sorption on CMBC600 and CMBC700. The competitive adsorption between OTC and CBL was also observed on the demineralized biochars (DCMBC600 and DCMBC700). For these biochars, an obvious decrease in the adsorption of both OTC and CBL was observed in the presence of a co-solute, with a more significant decrease determined for the OTC adsorption in the presence of CBL.

The strength of the competitive adsorption can be evaluated with a parameter $A,{ }^{38}$ which expresses the percentage decrease in $K_{\mathrm{oc}}$ following the addition of the co-solute and can be calculated using eqn (3):

$$
A=\left(K_{\mathrm{oc}}-K_{\mathrm{oc}}^{\prime}\right) / K_{\mathrm{oc}} \times 100 \%,
$$

where $K_{\mathrm{oc}}$ and $K_{\mathrm{oc}}^{\prime}$ are the sorption coefficients for the primary solute in the single-solute and bi-solute systems, respectively. The calculated values of $A$ for the adsorption of OTC and CBL on the original and demineralized biochars in binary-solute systems are shown and compared in Fig. 5. The OTC was found to be a much a stronger competitor to CBL than CBL was to OTC on the original biochars, as suggested by the 2.0-5.8 fold higher values of $A$ for OTC to CBL than CBL to OTC. In contrast, CBL exhibited a greater competition to OTC than OTC to CBL on the demineralized biochars, with 1.6-2.9 fold higher values of $A$ for addition of CBL to OTC than OTC to CBL.
The values of $n$ determined in the CBL sorption isotherms on original biochars in the presence of OTC increased significantly (from 0.382 to 0.845 for CMBC600, and from 0.282 to 0.667 for CMBC700, Table 3). In contrast, there was little change in the values of $n$ for OTC with CBL as the co-solute, implying that OTC can occupy and block certain sorption sites specific for CBL. ${ }^{30}$ For this reason, OTC showed stronger competitiveness relative

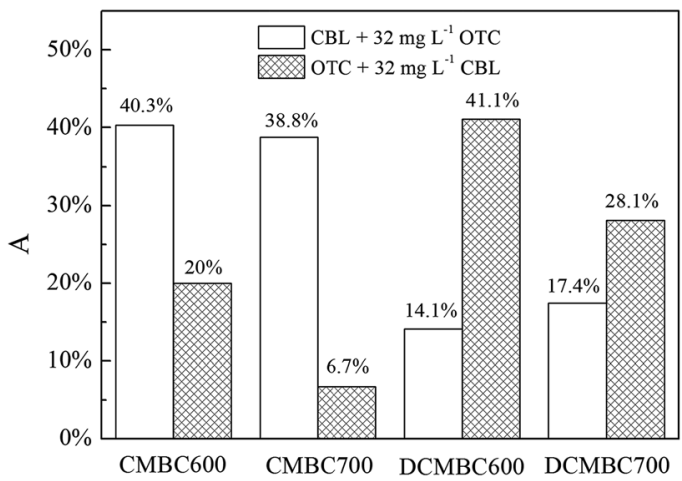

Fig. 5 The values of $A$ determined for the adsorption of carbaryl (CBL) and oxytetracycline (OTC) on original (CMBC600 and CMBC700) and demineralized (DCMBC600 and DCMBC700) biochars in the presence of OTC or CBL as the co-solute $\left(K_{\mathrm{oc}}\right.$ at $C_{\mathrm{e}}=0.05 \mathrm{~S}_{\mathrm{w}}$ for $\mathrm{CBL}, \mathrm{C}_{\mathrm{e}}=$ $0.2 \mathrm{mg} \mathrm{L}^{-1}$ for OTC). 
to CBL on CMBC600 and CMBC700. After demineralization, the changes in the values of $n$ in the CBL sorption isotherms with OTC as the co-solute were not as significant as those determined for OTC with CBL as the co-solute. A possible explanation for this observation is that the high-energy sorption sites offered by micropores are preferentially occupied by the smaller adsorbates (CBL) ${ }^{20}$ We have established that the main sorption mechanism for OTC on demineralized biochars is pore-filling. Therefore, CBL can occupy the micropores on demineralized biochars preferentially inducing a strong size-exclusion effect for the larger OTC molecules. Clearly, demineralization treatment changed the physicochemical properties of biochars and in turn altered the main sorption mechanisms for OTC and CBL on these materials, leading to changes in the competitiveness of OTC and CBL for binding sites. This observation, however, is not consistent with the sorption affinities-the values of $\log K_{\mathrm{oc}}$ for the CBL sorption isotherms were higher than those for the OTC on the original biochars, but at the same time lower than those of OTC on the demineralized biochars. In other words, a higher sorption does not always lead to a stronger competition. This phenomenon has also been observed in the study of Wang et al.,$^{38}$ where the sorption coefficients of sulfamethoxazole on activated carbon were found to be larger than those of sulfamethoxazole on single-walled carbon nanotubes. Yet, the sorption competition strength of sulfamethoxazole to carbamazepine was weaker on activated carbon than on single-walled carbon nanotubes in this study.

The results also reveal that the demineralization treatment weakened the competition strength of OTC to CBL, but enhanced that of CBL to OTC. As it can be seen from Fig. 5 that the values of $A$ for the competition of OTC to CBL on original biochars were 2.23-2.86 fold higher than those determined on the demineralized biochars. In contrast, the $A$ values of CBL to OTC on the original biochars were 2.06-4.19 fold lower than those determined for the demineralized biochars. This observation can be attributed to the different main mechanisms for the adsorption of OTC and CBL on demineralized biochars that we have established above. The sharp increase in SSA and the number of pore structures on the demineralized biochars offered abundant specific sites for CBL adsorption, thereby weakening the competitiveness of OTC to CBL following the demineralization treatment. Furthermore, as a result of its smaller size relative to OTC, CBL can take priority over OTC in occupying the micropores available on the demineralized biochars, and then markedly reduced the pore-filling effect of OTC. Ultimately, this phenomenon explains the greater competitiveness of CBL to OTC on demineralized biochars.

\section{Conclusions}

Mineral components of biochars affected both the adsorption and competitive adsorption of OTC and CBL on CMBCs and DCMBCs. Specifically, mineral component significantly enhanced the overall adsorption of CBL but inhibited slightly that of OTC to CMBCs, since the main sorption mechanisms of OTC and CBL after demineralization were altered to pore-filling and specific-site adsorption, respectively. The changes in the dominant sorption mechanisms for OTC and CBL led to their changes in competitive adsorption strength. Therefore, the impact of mineral component should be carefully considered when applying biochar as soil amendment to achieve efficient sorption of co-existed pesticides and antibiotics.

\section{Acknowledgements}

This study was financially supported by the National Natural Science Foundation of China (21577124), the National Basic Research Program of China (2014CB441103), and the National Science \& Technology Pillar Program of China (2015BAC02B01).

\section{References}

1 N. S. Bolan, D. C. Adriano and S. Mahimairaja, Environ. Sci. Technol., 2004, 34, 291-338.

2 B. Gámiz, R. Celis, C. H. María and J. Cornejo, J. Agric. Food Chem., 2010, 58(13), 7893-7901.

3 A. Karci and A. I. Balcioğlu, Sci. Total Environ., 2009, 407, 4652-4664.

4 A. B. A. Boxall, D. W. Kolpin, B. Halling-Sørensen and J. Tolls, Environ. Sci. Technol., 2003, 37, 286A-294A.

5 J. D. Martin, C. G. Crawford and S. J. Larson, National Water Quality Assessment Program, 2003, pp. 1992-2001.

6 M. X. Xie, W. Chen, Z. Y. Xu, S. R. Zheng and D. Q. Zhu, Environ. Pollut., 2014, 186, 187-194.

7 Y. P. Qiu, M. W. Wu, J. Jiang, L. Li and G. D. Sheng, Chemosphere, 2013, 93, 69-74.

8 X. H. Ren, P. Zhang, L. J. Zhao and H. W. Sun, Environ. Sci. Pollut. Res., 2016, 23, 2724-2734.

9 D. Mohan, A. Sarswat, Y. S. Ok and C. U. Pittman Jr, Bioresour. Technol., 2014, 160, 191-201.

10 M. Ahmad, A. U. Rajapaksha, J. E. Lim, M. Zhang, N. Bolan, D. Mohan, M. Vithanage, S. S. Lee and Y. S. Ok, Chemosphere, 2014, 99, 19-33.

11 D. Zhu and J. J. Pignatello, Environ. Sci. Technol., 2005, 39, 2033-2041.

12 R. Lohmann, J. K. MacFarlane and P. M. Gschwend, Environ. Sci. Technol., 2005, 39, 141-148.

13 Y. Chun, G. Y. Sheng, C. T. Chiou and B. S. Xing, Environ. Sci. Technol., 2004, 38, 4649-4655.

14 B. L. Chen, D. D. Zhou and L. Z. Zhu, Environ. Sci. Technol., 2008, 42, 5137-5143.

15 P. Zhang, H. W. Sun, L. Yu and T. H. Sun, J. Hazard. Mater., 2013, 244-245, 217-224.

16 M. Teixido, J. J. Pignatello, J. L. Beltran, M. Granados and J. Peccia, Environ. Sci. Technol., 2011, 45, 10020-10027.

17 M. Y. Jia, F. Wang, Y. R. Bian, X. Jin, Y. Song, O. F. Kengara, R. K. Xu and X. Jiang, Bioresour. Technol., 2013, 136, 87-93.

18 D. Zhu, S. Kwon and J. J. Pignatello, Environ. Sci. Technol., 2005, 39, 3990-3998.

19 X. D. Cao and W. Harris, Bioresour. Technol., 2010, 101, 52225228.

20 L. L. Ji, Y. Q. Wan, S. R. Zheng and D. Q. Zhu, Environ. Sci. Technol., 2011, 45, 5580-5586. 
21 K. Sun, M. J. Kang, Z. Y. Zhang, J. Jin, Z. Y. Wang, Z. Z. Pan, D. Y. Xu, F. C. Wu and B. S. Xing, Environ. Sci. Technol., 2013, 47, 11473-11481.

22 X. Y. Xu, X. D. Cao, L. Zhao, H. J. Zhou and Q. S. Luo, RSC Adv., 2014, 4, 44930-44937.

23 J. J. Pignatello, S. Kwon and Y. F. Lu, Environ. Sci. Technol., 2006, 40, 7757-7763.

24 C. Lattao, X. Y. Cao, J. D. Mao, K. Schmidt-Rohr and J. J. Pignatello, Environ. Sci. Technol., 2014, 48, 4790-4798.

25 H. Zheng, Z. Y. Wang, J. Zhao, S. Herbert and B. S. Xing, Environ. Pollut., 2013, 181, 60-67.

26 M. F. D. Oliveira, C. T. Johnston, G. S. Premachandra, B. Teppen, H. Li, D. Zhu and S. A. Boyd, Environ. Sci. Technol., 2005, 39, 9123-9129.

27 Y. Yang, L. Shu, X. L. Wang, B. S. Xing and S. Tao, Environ. Sci. Technol., 2011, 45(9), 3996-4002.

28 C. Moreno-Castilla, Carbon, 2004, 42, 83-94.

29 P. Kulshrestha, R. F. Giese and D. S. Aga, Environ. Sci. Technol., 2004, 38, 4097-4105.

30 S. A. Sassman and L. S. Lee, Environ. Sci. Technol., 2005, 39, 7452-7459.

31 A. Demirbas, Sci. Total Environ., 1998, 220, 235-241.
32 H. Schmitt, K. Stoob, G. Hamscher, E. Smit and W. Seinen, Microb. Ecol., 2006, 51, 267-276.

33 Y. N. Yang and G. Y. Sheng, Environ. Sci. Technol., 2003, 37, 3635-3639.

34 M. Keiluweit, P. S. Nico, M. G. Johnson and M. Kleber, Environ. Sci. Technol., 2010, 44, 1247-1253.

35 J. J. Pignatello and B. S. Xing, Environ. Sci. Technol., 1996, 30, 1-11.

36 L. Ji, W. Chen, L. Duan and D. Zhu, Environ. Sci. Technol., 2009, 43, 2322-2327.

37 M. Franz, H. A. Arafat and N. G. Pinto, Carbon, 2000, 38, 1807-1819.

38 C. Wang, H. Li, S. H. Liao, D. Zhang, M. Wu, B. Pan and B. S. Xing, Environ. Pollut., 2014, 194, 203-209.

39 S. Marie, W. W. C. Pascal and S. Mohamed, J. Soils Sediments, 2015, 15, 401-409.

40 USEPA and US Environmental Protection Agency, Health and Environmental Effects Profile for Captan, EPA/600/x-84/253, Environmental Criteria and Assessment Office, Office of Health and Environmental Assessment, Office of Research and Development, Cincinnati, OH, 1984.

41 C. Scherdel, G. Reichenauer and M. Wiener, Microporous Mesoporous Mater., 2010, 132(3), 572-575. 